\title{
Validation of a Passive Dynamic Walker Model for Human Gait Analysis
}

\author{
Ismet Handžić and Kyle B. Reed \\ Department of Mechanical Engineering \\ University of South Florida \\ Tampa, FL, 33620, U.S.A.
}

\begin{abstract}
While the dynamics and mathematics of passive dynamic walking (PDW) models have been extensively researched, it has not been until recently that they have been used for practical applications in rehabilitation and gait analysis. In this study, we evaluate the validity of using a two dimensional PDW for human gait analysis. Here, a PDW model is compared to recorded kinematic and kinetic walking data under normal conditions. We also study asymmetric gait by imposing a shank mass asymmetry and comparing it to a PDW model under the same asymmetric conditions. Kinematic and kinetic data for normal gait was taken from one subject using a motion capture system and a force plate, respectively. Gait under the asymmetric shank mass conditions was recorded by measuring the drifting radius of curvature from five participants walking blindfolded with a mass attached to their shank. While the PDW model lacks ankles (dorsiflexion), joint damping, and joint stiffness, the kinematics, kinetics, and gait asymmetry were comparable. Kinetic comparisons show agreement in general ground reaction force magnitude and profile. Kinematic results yield a good match in temporal and spatial gait characteristics. Asymmetric analysis of gait demonstrated that the PDW model can accurately predict the direction of step asymmetry.
\end{abstract}

\section{INTRODUCTION}

In this paper, we compare the kinematics, kinetics, and asymmetric behavior of a passive dynamic walker (PDW) to human gait. A PDW is a mechanical biped that walks down an inclined slope solely by the force of gravity. In contrast to humanoid robots, it shows a similar repeatable human-like gait [1]. A useful characteristic of PDWs is that a PDW model focuses on the passive dynamics of gait, excluding the cognitive controls of subject testing, so the purely dynamic aspects of gait can be analyzed. Here, we will directly compare PDW and human gaits.

For a rational comparison, the PDW model parameters are extracted from an anthropomorphic model [2] and proportionally sized such that the masses and mass distributions are scaled down from an average adult person (Figure 1). We define upper body to include the head, neck, and trunk without the arms. While this walking model has limitations, such as the lack of dorsiflexion (ankle movement in the sagittal plane), joint stiffness, and joint damping, it can give further insight into gait rehabilitation methods by modeling kinematic and kinetic gait characteristics.

First, we focus on explaining our PDW computer model, structure, and parameters. Then we compare and contrast kinematic and kinetic data of a normal, undisturbed, steady state, and symmetric PDW gait to a recorded normal human gait. Finally, we compare the gait of an asymmetric PDW to human gait with a weight attached to one shank.

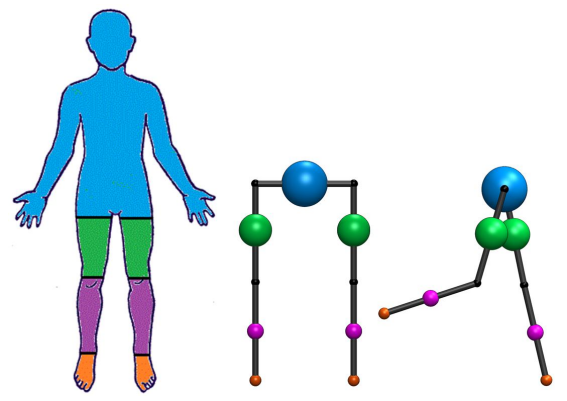

Fig. 1. PDW model used in this study based on an anthropomorphic model.

\section{BACKGROUND}

\section{A. Normal Human Walking}

In healthy human gait, one stride is the sum of a left and right step, where the two steps are symmetric. Each stride is divided into two distinct phases: the stance phase (foot is in contact with the ground), and the swing phase (foot swings by the hip). The swing phase is initiated by the toe-off (preswing) and terminated by the heel strike (initial contact). As the foot approaches heel strike, the knee locks (knee strike) to a stiff straight pose with a mean knee posture of around $5^{\circ}$ flexion; however subsequent to toe-off, knee flexion can reach up to $60^{\circ}$ [3]. The joints in human anatomy have stiffness and damping. Also, a normal human gait cycle includes a double support phase during which both feet are simultaneously on the ground. Double support accounts for approximately $10 \%$ of the gait cycle [3].

\section{B. Passive Dynamic Walking}

A PDW is an entirely mechanical device that is able to exhibit a steady and stable gait down an inclined slope purely due to gravitational forces and no other energy input. The energy gained by its progression down a slope due to gravity is lost during two inelastic collision events: the knee strike and the heel strike; hence, it is completely passive.

For decades, PDWs have been constructed and mathematically analyzed [5][6][7][4], ameliorating their physical designs and analytical models. The two-dimensional PDW concept used for our analysis was pioneered by analyzing a rimless wheel progressing down a slope [1], then developed into a double pendulum model [6], and a kneed walker [7]. By differentiating left and right legs and varying leg mass and mass distribution, Honeycutt et al. [4] enabled an asymmetric PDW. However, only in recent years have these devices been put into use for more practical applications such as an actuated PDW to compensate for gravity [8], a steering PDW [9], and prosthesis design [10]. 
TABLE I

HUMAN AND PDW MODEL PARAMETERS USED FOR MODEL VALIDATION AND EXPERIMENTAL COMPARISON.

\begin{tabular}{|c|l|l|l|l|l|l|}
\hline & \multicolumn{2}{|l|}{$\begin{array}{l}\text { Anthropomorphic Model Mass } \\
\text { for Person (kg) }\end{array}$} & \multicolumn{2}{|l|}{$\begin{array}{l}\text { Anthropomorphic Model Mass } \\
\text { for PDW model (kg) }\end{array}$} & \multicolumn{2}{l|}{$\begin{array}{l}\text { Anthropomorphic Model Segment } \\
\text { COM Distance From Hip (m) }\end{array}$} \\
\hline & Human & \% Total & Human & \% Total & Human & Model \\
\hline Upper Body & 52.90 & $58.32 \%$ & 4.00 & $57.31 \%$ & 0.00 & 0.00 \\
Thigh & 24.40 & $26.90 \%$ & 1.90 & $27.22 \%$ & 0.22 & 0.22 \\
Knee & 0.00 & $0.00 \%$ & 0.00 & $0.00 \%$ & 0.50 & 0.49 \\
Shank & 9.23 & $10.18 \%$ & 0.60 & $8.60 \%$ & 0.72 & 0.72 \\
Foot & 2.91 & $3.21 \%$ & 0.22 & $3.15 \%$ & 0.98 & 1.00 \\
Full Body & 90.7 & $100.00 \%$ & 6.98 & $100.00 \%$ & - & - \\
\hline
\end{tabular}

An attractive aspect of a PDW to the field of gait analysis is that it allows us to separate the purely mechanical attributes of walking from the cognitive controls of the human body. This characteristic is advantageous when it is desired only to study the physical parameters of human gait. All humanoid robots either follow a quasi-static pattern and/or require controllers to model the feedback law. Neither approach is analogous to human gait since human walking is dynamically stable and robot controllers do not yet adequately model the human sensorimotor system. A dynamically stable passive model is more realistic of the natural human gait dynamics and can predict the motions from altered dynamics.

\section{PDW MODEL DESCRIPTION}

The model is a $2 \mathrm{D}$ passive multi-pendulum system in constant contact with the ground. The dynamics can be described with the Lagrangian formulation of a multipendulum system [7][4]. The collision events are modeled as instantaneous inelastic collision events and post collision velocities are calculated using conservation of angular momentum. This model however does not account for dorsiflexion, joint stiffness, and joint damping. This model also does not exhibit a double support phase during walking. However, despite these limitations, the model captures certain aspects of gait. We are also able to easily change model parameters such as limb length, masses, and mass distribution, all of which affect the PDW gait stability and symmetry, some of which are difficult to change in humans.

\section{A. Seven Mass Model}

Honeycutt et al. [4] describe a nine mass PDW model with a hip mass, two thigh masses on each leg, and two shank masses on each leg. Due to the nature of our research here, we only employed seven of the nine masses, setting two thigh masses to zero and moving the lower shank mass down to represent a foot mass. As shown in Figure 1, the seven mass model relates directly to the trunk, thigh, shank, and foot masses.

\section{B. Model Scaling}

The objective of this paper is to validate and present insight into using a PDW for human gait analysis. Therefore, it is important to closely match and properly scale the PDW model to an actual individual. For correct scaling, we reference available anthropomorphic data [2], which outlines average masses and mass distributions for a human upper body, thigh, shank, and foot. According to Perry et al. [3], the upper body travels as a unit during normal gait and only moves up and down; hence the upper body, which includes the head, neck, and trunk, is represented in our PDW model as only one hip mass. Arms are excluded here, but are typically only included in physical PDWs to prevent twisting.

Our PDW model is one meter in height (ground to hip) while the hip height for the tested individuals is also approximately one meter. In conjunction with the anthropomorphic data, this yielded a scaled down mass distribution between the tested individuals and PDW model, scaling the thigh, shank, and foot masses accordingly. Table I shows the masses and mass distribution of actual subject measurements for normal walking and corresponding PDW model values. All center of mass distributions for the human and the PDW adhere to the anthropomorphic model.

\section{Model Criteria}

For the model comparison and experiment, steady state measurements were taken of a stable walking model for normal walking. Our stability criteria was defined as fifty steps without the PDW tumbling or with foot drag. To ensure steady state, the PDW model results were taken during stride number thirty-five and thirty-eight, at which point all trials reached asymptotic stability. During each trial the position and velocity of the PDW heel and hip mass were recorded. All PDW data is in the sagittal plane. The PDW ramp angle was $3.2^{\circ}$ for all trials because it was found to be the most stable decline value.

\section{HumAN EXPERIMENT DATA}

All seven participants read and signed a consent form approved by the University of South Florida's IRB prior to participating in the three experiment types below.

\section{A. Normal Gait Kinetics}

For comparison, human walking kinetics were recorded from one healthy subject (male, age $32,1.83 \mathrm{~m}, 71 \mathrm{~kg}$ ) walking over a force plate seven times. Horizontal and vertical ground reaction forces were recorded during the stance phase during steady state walking. This data was recorded using a AMTI model OR6-5 biomechanics platform. Figure 2a presents this recorded kinetics data.

\section{B. Normal Gait Kinematics}

Human walking kinematics were recorded from one healthy subject (male, age $26,1.86 \mathrm{~m}, 90.7 \mathrm{~kg}$ ) by a 3D VICON motion capture system infrared camera at $120 \mathrm{~Hz}$ in the sagittal plane, capturing strides three and four out of 


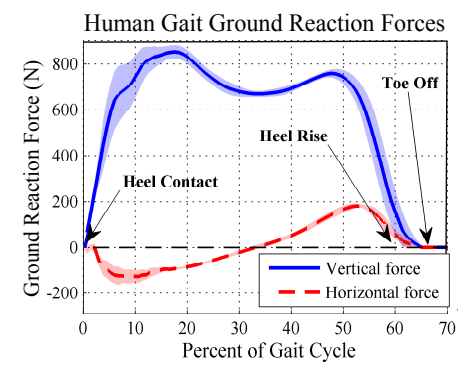

(a)

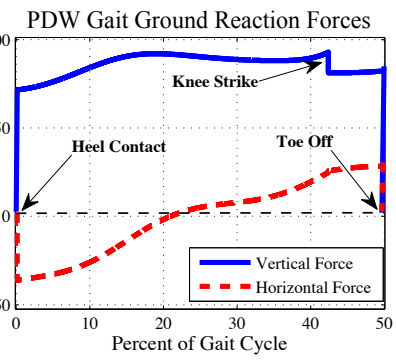

(b)
Fig. 2. Comparison of kinetics in (a) a human and (b) the modeled PDW gait during stance phase. Shading represents 1 standard deviation in the human data; there was no variation in the PDW model.

five total. The recorded individual had markers placed on their knees, left and right ilium of the hip bone, and on the lowest point on the back of the subject's sneaker of both feet. Figure 3 a presents this recorded kinematics data.

\section{Asymmetric Gait}

Five participants (age $22 \pm 1.9,1.74 \pm 0.06 \mathrm{~m}, 88 \pm 13 \mathrm{~kg}$ ) were asked to walk with and without a mass attached to their shank. First, each participant walked normally with a blindfold and no mass attached. By blindfolding the participant, visual feedback was removed. They walked $9.1 \mathrm{~m}$ where the deviation from the straight forward path was measured every $2.3 \mathrm{~m}$ by laying markers down as they crossed each discrete distance, shown in Figure 4 a.

Next, the participants walked wearing a blindfold twice more in the same fashion; however this time with a $4.54 \mathrm{~kg}$ mass firmly strapped to the middle of their right shank. To trace the participants' walking trajectories, the deviation from the straight forward path was measured discretely. After each trial, the weight was removed from the participant's leg in order to minimize gait adaptation. To further prevent adaptation, the blindfolded participant was told that each trial may use a different weight.

\section{RESUlTS AND COMPARISONS}

\section{A. Normal Gait Kinetics}

Considering the limitations of the PDW model, the gait kinetics of the PDW and human are very comparable. As shown in Figure 2, the vertical forces peak and valley during the mid-stance phase at similar instances, but the magnitudes are different. The exaggerated shape of the human is likely caused by the model's lack of dorsiflexion. The smooth initial and final change in the human walking is not present in the PDW model.

The PDW model and human horizontal reaction forces switch from resisting to assisting forward progression at the same time during stance. However, the maximum forces are slightly different. The PDW model's horizontal reaction forces have a maximum backward force of $48 \%$ of the walker mass at heel contact and a maximum forward force of $37 \%$ of the walker mass at toe off. The human data shows smaller forces: maximum backward force is $23 \%$ of the body mass at $8 \%$ of the gait cycle and the forward force is $26 \%$ of the body mass at $53 \%$ of the gait cycle.
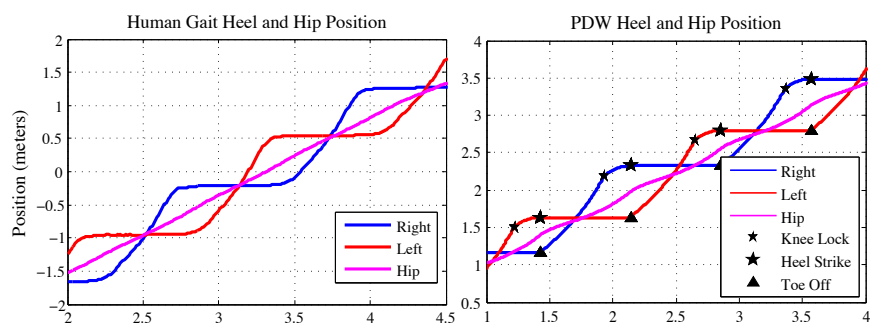

Human Gait Heel and Hip Position

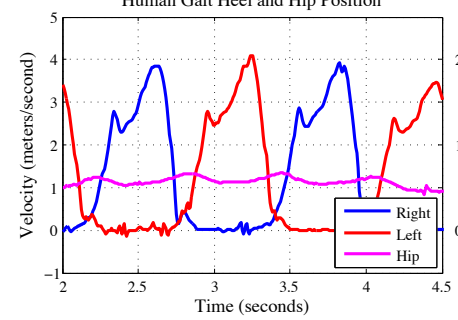

(a)

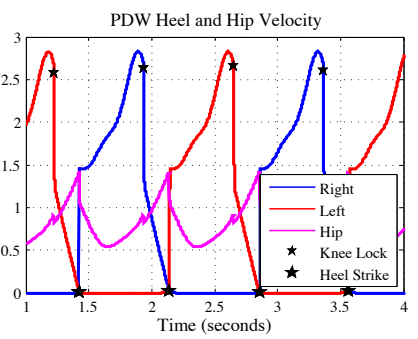

(b)
Fig. 3. (a) Human and (b) PDW walking. While similar in general kinematics, differences arise due to the lack of double support and dorsiflexion in the PDW model.

It is apparent that the lack of double support and muscle energy storage/release in the PDW model creates differences. As the PDW engages heel contact and toe off, ground reaction forces instantaneously change, while human walking gradually transitions. This abrupt change in the PDW is due to the absence of dorsiflexion of the opposite foot and double support phase, both of which smooth out the switching in human walking. Also, the collision events are modeled as an inelastic collision, so the knee strike becomes apparent as a drop in vertical forces and a leveling of horizontal ground reaction forces. This is not evident in normal walking kinetics due to joint stiffness and joint damping.

\section{B. Normal Gait Kinematics}

As observed in Figure 3, both human and PDW model kinematics have rhythmic, repetitive, and symmetric motions; however the foot velocity of the scaled down model is $83 \%$ of the human. This difference in velocity magnitude is due to the PDW's passive nature in that it has no muscle action to propel it forward and only relies on gravity.

While fluctuations of the hip's velocity profile happen during the same instances in the human and model gait cycles, the model's hip velocity profile is more exaggerated: $40 \%$ variation for the model and $10 \%$ for the human. This higher fluctuation is explained by the PDW movement down a ramp and the participant's dorsiflexion, which softens the gait. Unlike horizontal walking, the PDW hip drops the extra distance down the ramp prior to heel strike. Also, during the gait cycle in human walking the ankle flexes (dorsiflexion) right before toe off (storing elastic energy) and recoils during toe off (dissipating energy); this movement creates a smoother transition between stance and swing phase.

During stance phase, as the foot approaches knee strike, the human data has a slight dip in velocity while the model velocity at the same instant has a minor bump with a steady increase until knee strike. The velocity dip in human walking is caused by dorsiflexion of the ankle in humans when the 
tibialis anterior (frontal shank) muscles contract, while the posterior shank muscle group stretches, again storing and releasing energy for a smoother transition [3].

Both human and model temporal and spacial characteristics such as gait cycle duration, stride length, and walking velocity are in good comparison. Table II shows these characteristics, including data found in the literature [11] taken from eleven subjects.

TABLE II

PDW Model Versus Human Average Walking

\begin{tabular}{|c|l|l|l|}
\hline & PDW Model & Measured & $\begin{array}{l}\text { McIntosh } \\
\text { et. al. [11] }\end{array}$ \\
\hline Gait Cycle Time (s) & $1.42 \pm 0.01$ & $1.24 \pm 0.1$ & $1.0 \pm 0.1$ \\
Stride Length (m) & $1.17 \pm 0.001$ & $1.46 \pm 0.02$ & $1.41 \pm 0.08$ \\
Walking Velocity (m/s) & $0.82 \pm 0.24$ & $0.98 \pm 0.37$ & $1.57 \pm 0.12$ \\
\hline
\end{tabular}

\section{Asymmetric Gait}

This analysis examines walking patterns with asymmetric mass loading. Increasing the right shank mass yielded an increased right step length naturally causing the individuals to veer to the left (Figure 4). Participants veered to the left $9.3 \pm 3.8 \%$ with a shank mass that was $5.26 \pm 0.8 \%$ body mass.

In order to compare a two dimensional step asymmetry of the PDW walker, the deviation per stride was calculated using the mean participant hip width of $0.381 \mathrm{~m}$ and Equation 1.

$$
\frac{\text { Deviation }}{\text { Stride }}=\text { Stride Length } * \frac{\text { Step Asymmetry }}{\text { Hip Width }},
$$

The PDW was shown to be unstable with a properly scaled shank mass asymmetry matching the experiment. However, if the asymmetry mass is scaled down additionally by a factor of twenty $(0.26 \%$ total walker mass), the walker trajectory is within the bounds of experimental data. This deviation is compared to experimental data in Figure 4. Because the vestibular system in humans likely measures the trajectory change and the sensorimotor system compensates slightly to prevent drastic deviation from initial path, the PDW model is assumed to always deviate more due to its purely dynamic nature. Although the scale factor is off, the direction is still
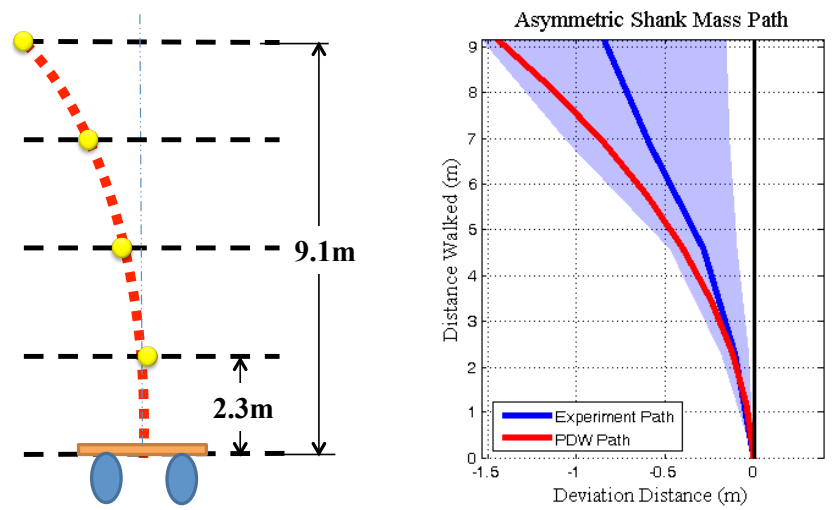

Fig. 4. Left: blindfolded the subject is asked to walk forward while deviation from initial trajectory path was recorded at discrete distances. Right: mean experimental trajectory compared to PDW Model. in agreement. Thus, the model can be used to predict general trends, but is unlikely to predict magnitudes.

\section{CONClusions And Future Work}

We have compared some aspects of gait and shown that the passive dynamic walker (PDW) can be a valuable research asset in analyzing the passive dynamics of gait, particularly when looking at how changing dynamics will affect gait patterns. The walker, sized with an anthropomorphic model, corresponds well to normal human walking. Although there are variations between human anatomy and our PDW model, such as the lack of dorsiflexion, joint stiffness, and joint damping, this PDW model can be used to approximate gait cycle time, step length, general kinematic trajectories of the hip and feet, reaction forces, and the direction of step asymmetry for limb mass asymmetry. Extending the PDW model to account for its current drawbacks by adding joint stiffness and joint damping will likely increase the agreement between model prediction and human measurements.

Future research can use the PDW model to examine additional changes in gait by altering the passive dynamics. For example, research could examine the asymmetric mass distribution and asymmetric limb length where the two changed parameters are applied to opposite feet; can they cancel out the asymmetric effects? In essence, making a symmetric gait with a physically asymmetric system.

\section{AUthor CONTRIBUtions}

I. Handžić was responsible for the idea generation, experiments, data analysis, and writing. S. Carey presented the research. K. Reed was responsible for idea generation, data analysis, and writing.

\section{REFERENCES}

[1] T. McGeer, Passive Dynamic Walking, The International Journal of Robotics Research, vol. 9, no. 2, pp. 62-82, 1990.

[2] R. Drillis et al. Body segment parameters: a survey of measurement techniques. National Academy of Sciences, 1964.

[3] J. Perry, Gait Analysis: Normal and pathological function, N. S. Inc., Ed. Thorofare, 1992, vol. 50.

[4] C. Honeycutt, J. Sushko, and K. B. Reed, Asymmetric passive dynamic walker, in Proc. IEEE Int. Conf. Rehabilitation Robotics (ICORR), pp. 852-857, 2011.

[5] M. Garcia, A. Chatterjee, A. Ruina, and M. Coleman, The simplest walking model: Stability, complexity, and scaling, Journal of Biomechanical Engineering, vol. 120, no. 2, p. 281288, 1998.

[6] A. Goswami, B. Thuilot, and B. Espiau, A Study of the Passive Gait of a Compass-Like Biped Robot, The International Journal of Robotics Research, vol. 17, no. 12, pp. 1282-1301, 1998.

[7] V. F. H. Chen, A Passive Dynamic Walking with Knees: A Point Foot Model, Master's thesis, Massachusetts Institute of Technology, 2005.

[8] R. D. Gregg. Controlled Reduction of a Five-Link 3D Biped with Unactuated Yaw, Proc. of the 5th Intl. Conf. on Decision and Control and European Control Conference (CDC-ECC), 2011.

[9] R. Gregg, A. Tilton, S. Candido, T. Bretl, and M. Spong. Control and Planning of 3-D Dynamic Walking With Asymptotically Stable Gait Primitives, IEEE Transactions on Robotics, vol. 28, no. 6, 2012.

[10] J. Sushko, C. Honeycutt, K. B. Reed, Prosthesis Design Based on an Asymmetric Passive Dynamic Walker, 4th Intl. Conf. on Biomedical Robotics and Biomechatronics (BioRob), pp. 1116-1121, 2012.

[11] A.S. McIntosh et. al. Gait Dynamics on an inclined walkway. Journal of Biomechanics. Vol. 39. pp 2491-2502. 2006

[12] B. Gurney. Leg Length Discrepancy. Gait and Posture. Vol. 15. pp 195-206. 2002 